\title{
Adding "colors" to cryo-EM: extracting local chemical data from radiation damage
}

Gili Abelya ${ }^{1}$, Leonard Joseph Campanello ${ }^{2}$, Ran Zalk ${ }^{3}$ and Gabriel A Frank ${ }^{1}$

${ }^{1}$ Dept. of Life Sciences, Ben-Gurion University of the Negev, United States, ${ }^{2} 205$ Orient St. Bayonne, NJ

07002, United States, ${ }^{3}$ Ilse Katz Institute for Nanoscale Science \& Technology, Ben-Gurion University of the Negev, United States

The harsh radiation inside the electron microscope leads to the gradual deterioration of the sample [1-2]. Organic molecules are typically more sensitive to radiation damage. For that reason, controlling the accumulated dose is crucial for high-resolution cryo-EM data collection [1] and post-acquisition analysis [3].

Different chemicals respond differently to the electron radiation. As a result, the progression of radiationinduced damage varies across the field of view, reflecting the sample's local chemical properties. Because cryo-EM data is collected as a series of dose fractionated frames (movies), the gradual radiation-induced deterioration of various chemical moieties in the sample is imprinted in all single-particle cryo-EM datasets. Analysis of this information can provide new insights into the structure and function of macromolecular complexes from already existing data.

This talk will cover our recent advances in extracting and analyzing the local sensitivity to radiation damage from single-particle cryo-EM data. Several methods for analyzing and interpreting local radiation damage sensitivities will be presented using examples from datasets of ferritin, protective antigen (the anthrax toxin's translocase), and the proteasome core particle. Analysis of local radiation sensitivities can guide the fitting of molecular models into the EM maps, disentangle the 3D organization of macromolecular complexes with mixed stoichiometries, and shed light on ligand binding.

As an initial step for analyzing the radiation damage progression, we developed a code for reconstructing a 3D map from each frame in single-particle datasets, generating 3D movies (Figure 1). To ensure accurate per-frame alignment, which is essential for attaining high-resolution maps, we based the reconstructions on Relions's particle polishing metadata.

Analysis of the radiation damage was used to assign unique values ("colors") to various regions in the 3D density maps. These colors were associated with the different amino acids and small ligands, thereby simplifying-model building by guiding the placement of amino acids inside the 3D maps (Figure 1b).

Oligomers with non-uniform stoichiometry of structurally similar subunits such as ferritins, which are a mix of two types of subunits, pose a challenge for current structural methods. The different radiation damage sensitivities of the amino acids in the different subunits can highlight the differences between them, thereby helping to determine their organization in the oligomer.

When the differences in radiation sensitivities are large, as is the case of complexes of minerals and biomineralization proteins, radiation damage analysis can be used to segment the densities of irregularly shaped ion clusters of inorganic sediments by direct comparison of different frames in the 3D movies and by dimensional reduction. We recently applied this analysis method for following the structural changes in an intrinsically disordered iron-binding domain of the magnetite mineralization protein Mms6 in response to its interaction with nascent sediment (Figure 2) [4]. 


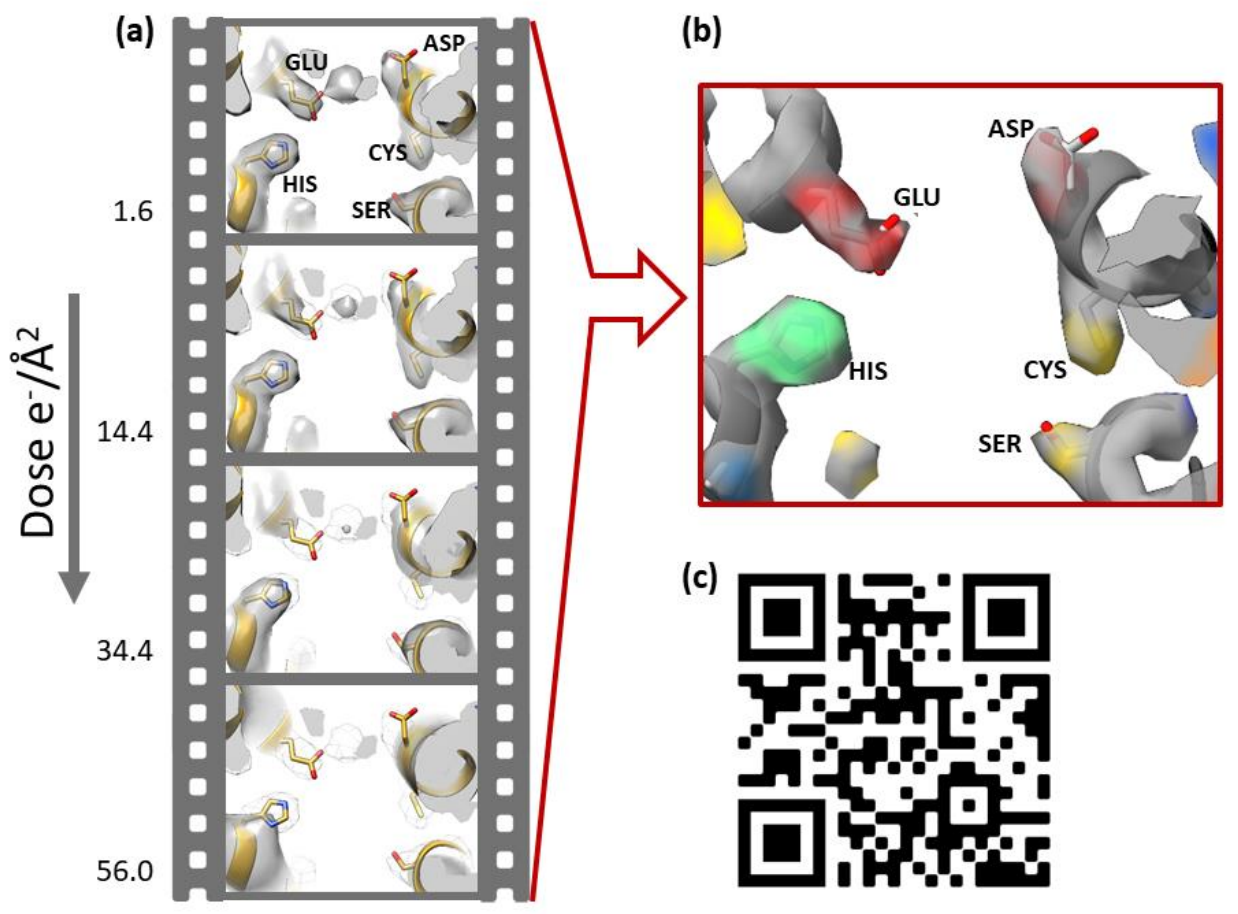

Figure 1. Figure 1: Coloring EM density maps by differences in radiation sensitivities. (a) Time-lapse frames in a 3D movie showing the gradual decay of the signal from different amino acids. Frames were taken from a cryo-EM dataset of L-ferritin. Each frame was reconstructed following our per-frame reconstruction protocol (modified from [4]). The densities of various amino acid decay as expected from their respective radiation sensitivity [1]: GLU, ASP > CYS, SER > HIS. (b) the same region as in (a) collared by radiation damage sensitivity (GLU and ASP - red, CYS, SER - yellow, HIS - green). (c) See also the movie: https://youtu.be/Lv7Y6_MgVws. The accumulated dose is in (e-/A2) is indicated to the left of each frame. 


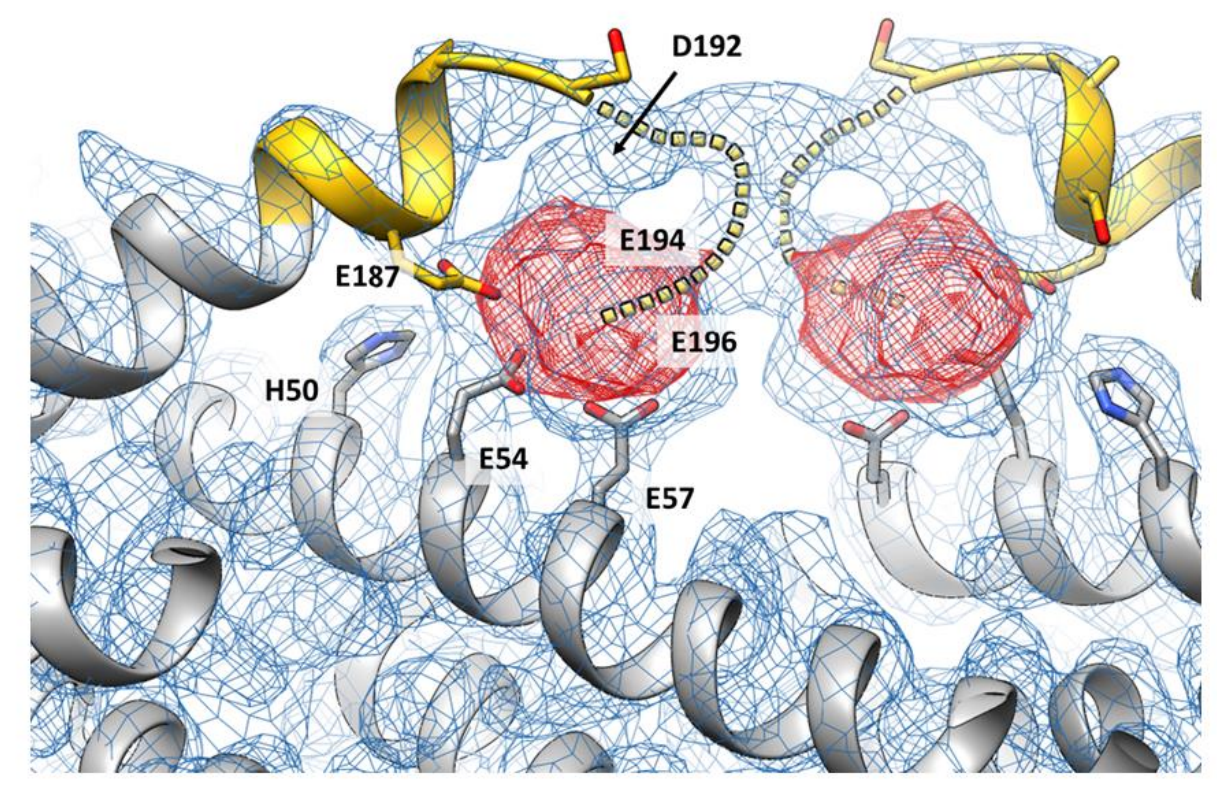

Figure 2. Figure 2: Segmentation of an iron-oxide/protein complex by radiation damage analysis (adapted from [4]). The EM density map of an iron-binding peptide fused to the inner surface and a nascent sediment were reconstructed twice; once as "regular" dose weighted map (yellow mesh) and once only with the late frames (red mesh). The late frames-map shows only the radiation resilient inorganic sediment. The molecular model of the peptide and the ferritin is presented as ribbon representation (yellow and gray) respectively.

\section{References}

(1) Baker, L. A.; Rubinstein, J. L. Radiation Damage in Electron Cryomicroscopy; Elsevier Masson SAS, 2010; Vol. 481. https://doi.org/10.1016/S0076-6879(10)81015-8.

(2) Hattne, J.; Shi, D.; Glynn, C.; Martynowycz, M. W.; Rodriguez, J. A.; Gonen Correspondence, T. Analysis of Global and Site-Specific Radiation Damage in Cryo-EM. Structure 2018, 26, 759-766. https://doi.org/10.1016/j.str.2018.03.021.

(3) Zivanov, J.; Nakane, T.; Forsberg, B.; Kimanius, D.; Hagen, W. J. H.; Lindahl, E.; Scheres, S. H. W. RELION-3: New Tools for Automated High-Resolution Cryo-EM Structure Determination. bioRxiv 2018, 1-22. https://doi.org/10.1101/421123.

(4) Davidov, G.; Abelya, G.; Zalk, R.; Izbicki, B.; Shaibi, S.; Spektor, L.; Shagidov, D.; Meyron-Holtz, E. G.; Zarivach, R.; Frank, G. A. Folding of an Intrinsically Disordered Iron-Binding Peptide in Response to Sedimentation Revealed by Cryo-EM. Journal of the American Chemical Society 2020, 142 (46), 19551-19557. https://doi.org/10.1021/jacs.0c07565. 